\title{
Trans-National Equitable Strategies of Vaccine Distribution during the COVID-19 Pandemic
}

\author{
D. Domingo ${ }^{1} \dagger$, S. Biber ${ }^{2}$, G. Dian ${ }^{1}$, P. Dorey ${ }^{1}$, K. Haddad $^{3}$, P. Heslop $^{1}$,

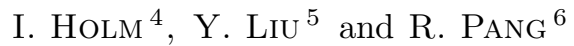 \\ ${ }^{1}$ Department of Mathematical Sciences, Durham University, UK \\ ${ }^{2}$ Department of Engineering Mathematics, University of Bristol, UK \\ ${ }^{3}$ Niels Bohr Institute, University of Copenhagen, Denmark \\ ${ }^{4}$ Institut de Physique Théorique, Université Paris-Saclay, France \\ ${ }^{5}$ Mathematical Institute, University of Oxford, UK \\ ${ }^{6}$ Department of Mathematics, The London School of Economics and Political Science, UK
}

(Communicated to MIIR on 14 October 2021)

Study Group: ESGI 165, Durham, UK, 12th-16th April 2021.

Communicated by: Bernard Piette

Industrial Partner: Various UK Government departments via the Virtual Forum for Knowledge Exchange in Mathematical Sciences (V-KEMS).

Presenter: Guven Demirel on behalf of V-KEMS, g.demirel@qmul.ac.uk.

Team Members: S. Biber, University of Bristol; A. Champneys, University of Bristol; G. Dian, Durham University; D. Domingo, Durham University; P. Dorey, Durham University; K. Haddad, Niels Bohr Institute; P. Heslop, Durham University; I. Holm, Université Paris-Saclay; Y. Liu, University of Oxford; R. Pang, LSE.

Industrial Sector: Government.

Key Words: Vaccination Strategy; Social/Vaccination Cost; Trans-national Vaccine Sharing; COVID-19.

MSC2020 Codes: 34C60; 65L05; 90C26; 91B32.

$\dagger$ Report Editor: dario.domingo@durham.ac.uk 


\section{Summary}

In this report we propose a modelling framework to analyse and optimise worldwide vaccine allocation strategies, with reference to the current COVID-19 pandemic. We model epidemiological transmission and vaccination in a system of $M$ interacting countries, quantifying the social and economic costs incurred by each country due to the pandemic and the country's vaccination rate. Under constraints over global vaccine availability, we analyse best strategies of vaccine allocation: either with the aim of minimising global costs, or by taking the perspective of a competitive game where each country aims to minimise its own cost. We distinguish between the financial capabilities of different countries, and extend our framework to allow for vaccine donation from wealthier to poorer countries. Numerical simulations allow us to compare the best strategies of the above two approaches, and to analyse circumstances under which vaccine donation simultaneously benefits both donating and receiving countries.

\section{Introduction}

The COVID-19 pandemic has drastically affected life across the globe since the novel coronavirus, later named SARS-CoV-2, was first discovered in December 2019. The human and economic costs of the pandemic have been immense: according to the World Health Organization, there have been over 151 million confirmed cases and over 3.1 million deaths globally as of May 4th, 2021 [1] - staggering figures which certainly underestimate the actual spread of the virus. Moreover, the pandemic strongly impacting the world economy, the magnitude of which is estimated to be on the order of tens of trillions of dollars [2]. Despite the magnitude of devastation brought about by this virus, there is light at the end of the tunnel. Safe and effective vaccines against the virus have been developed, tested, and approved for use at impressive speed [3], and vaccination efforts are underway around the world.

Immunising populations through the deployment of vaccines represents the clearest and safest strategy out of the pandemic. Experts warn, however, that a hoarding of vaccines by wealthy nations will only prolong the pandemic, and may result in greater long-term losses even for those wealthy nations $[4,5]$. A continued spreading of the virus in countries that cannot afford to vaccinate their residents is clearly an undesirable prospect for any such country. It also poses a persistent threat to global health by risking the emergence of viral variants that are unaffected by present vaccines [6]. Thus, a complete COVID19 recovery strategy will require global cooperation, and must promote the equitable distribution of vaccines amongst all countries in place of nationalistic approaches.

In this report we present the results of the collaborative work carried out by the authors during the European Study Group with Industry 165, in which we lay the conceptual and mathematical foundations of a vaccine allocation model. The ultimate goal of the model is to suggest strategies that minimise global losses and to investigate the potential advantage of having wealthier nations donate part of their vaccines to countries in need. We approach the problem by making use of a Susceptible-Infected-Recovered (SIR) model, 
suitably modified to include a vaccination compartment. The full model is thus dubbed a Susceptible-Infected-Recovered-Vaccinated - or SIRV - model. The coexistence in the model of epidemiological and vaccination indices allows us to define a cost function for each country, which includes both social and economical components. The parameters in the cost function provide a means for differentiating between wealthy and poor nations, and for extending the framework to include the possibility of vaccine donations from wealthier to poorer nations. We thus investigate optimal strategies for worldwide vaccination using two different approaches: one where costs are minimised globally, and one where each country attempts to minimise its own costs, as in a competitive game. Similarities and differences between the results of the two approaches are discussed.

The report is organised as follow. In section 2 we introduce the set of coupled differential equations describing the SIRV model. We then discuss the form of our cost functions in section 3 and extend the framework to allow for vaccine donation in section 4 . The first of our minimisation approaches (minimising global costs) is discussed in section 5 , while the game theoretic perspective is expanded upon in section 6 . Numerical simulations are shown throughout. Finally, we conclude and discuss future directions of study in section 7 .

\section{The SIRV Model}

As discussed in the Introduction, our aim is to model the impact of vaccination on an ongoing pandemic, such as the current COVID-19 one, and to investigate international "optimal" strategies of vaccine distribution. To achieve the above aims we make use of an epidemiological compartmental model. We describe this in subsection 2.1 and discuss choices of its parameters in subsection 2.2.

\subsection{Modelling Epidemiological Transmission and Vaccination}

Consider a total of $M$ countries, each with population size $N_{i}, i=1, \ldots, M$. Before introducing vaccination, we draw from the classical idea of an SIR model and distinguish between the following three types of individuals: susceptible, who have never caught the virus; infected/infectious, who are currently infected and can spread the disease to susceptible individuals; recovered/removed, who have had the disease and have either recovered from it or passed away. For $i=1, \ldots, M$, we therefore define the following variables:

- $S_{i}(t)$ : number of susceptible people in country $i$, at time $t$;

- $I_{i}(t)$ : number of infected/infectious people in country $i$, at time $t$;

- $R_{i}(t)$ : number of recovered/removed people in country $i$, at time $t$.

Susceptible individuals may become infected, and infected ones may recover. The rate at which infected individuals of country $j$ infect susceptible individuals of country $i$ is denoted by $\beta_{i j} \geq 0$ : more precisely, $\beta_{i j}$ is the average number of susceptible individuals of country $i$ which one infected person in country $j$ infects over the course of one day. The rate at which infected individuals recover is instead denoted by $\gamma$, in all countries. 
This leads to the following SIR model:

$$
\begin{aligned}
\frac{d S_{i}}{d t} & =-\sum_{j} \beta_{i j} I_{j} \frac{S_{i}}{N_{i}}, \\
\frac{d I_{i}}{d t} & =\sum_{j} \beta_{i j} I_{j} \frac{S_{i}}{N_{i}}-\gamma I_{i}, \\
\frac{d R_{i}}{d t} & =\gamma I_{i},
\end{aligned}
$$

where the total population of each country remains constant over time: $S_{i}+I_{i}+R_{i}=N_{i}$. As noted at the beginning, this does not include vaccination yet. To model vaccine rollout, we include the following variable:

- $V_{i}(t)$ : number of people in country $i$ that have been vaccinated by time $t$.

We denote by $\xi_{i}(t)$ the rate at which vaccines are administered to the population in country $i$, hence $V_{i}(t)=\int_{0}^{t} \xi_{i}(s) d s$. Our model assumes that vaccines are administered to any individual not yet vaccinated, rather than to susceptible individuals only (justification below). Thus, by allocating the rate $\xi_{i}(t)$ to the three compartments $S_{i}, I_{i}, R_{i}$, proportionally to their size, from model (2.1) we get the following:

$$
\begin{aligned}
\frac{d S_{i}}{d t} & =-\sum_{j} \beta_{i j} I_{j} \frac{S_{i}}{N_{i}}-\frac{S_{i}}{S_{i}+I_{i}+R_{i}} \xi_{i}(t), \\
\frac{d I_{i}}{d t} & =\sum_{j} \beta_{i j} I_{j} \frac{S_{i}}{N_{i}}-\gamma I_{i}-\frac{I_{i}}{S_{i}+I_{i}+R_{i}} \xi_{i}(t), \\
\frac{d R_{i}}{d t} & =\gamma I_{i}-\frac{R_{i}}{S_{i}+I_{i}+R_{i}} \xi_{i}(t), \\
\frac{d V_{i}}{d t} & =\xi_{i}(t),
\end{aligned}
$$

where $S_{i}(t)+I_{i}(t)+R_{i}(t)+V_{i}(t)=N_{i}$ for all $t$. We refer to model (2.2) as to the SIRV model. This is the set of $4 M$ coupled ODEs we use to model simultaneously the spread of the epidemic and the vaccine rollout in the populations of $M$ interacting countries. The flow of individuals from one compartment to the other of a single country, and the associated rates, are illustrated in Figure 1.

The question of which classes of people should be vaccinated in our model was discussed during the study group. Current policies in this regard vary from country to country. Several of them - such as the USA, the UK, Italy, France and many more, see for example [7], [8] - currently vaccinate individuals who have recovered from a previous infection, often with one dose in place of two where relevant, see [9]. Individuals known to be infected are instead rarely vaccinated. However, some fraction of unknowingly infected individuals is inevitably vaccinated while infected. Indeed, [10] estimates the percentage of entirely asymptomatic infections to be about $15-20 \%$, the estimate rising up to $50 \%$ if the diagnosis is made at an early stage of infection. In light of this, and to keep complexity to a reasonable level during the 4-day study group collaboration, we decided to consider a model where not only susceptible, but also infected and recovered individuals 


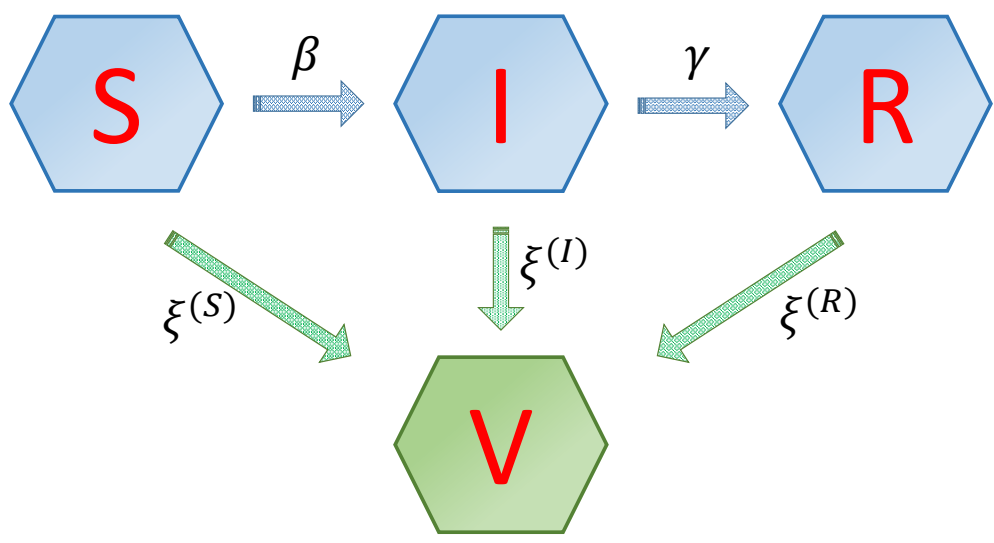

Figure 1. Visual representation of the compartmental model described in section 2, for a single country. Arrow labels denote transition rates between compartments (Susceptible, Infected, Recovered, Vaccinated). For each $j=S, I, R, \xi^{(j)}=j /(S+I+R) \times \xi$, where $\xi$ is the country's vaccination rate.

are offered the vaccine. More involved ideas, such as vaccinating only a fraction of infected individuals, are left for future work.

\subsection{Parameter Estimation and Numerical Simulations}

Since the onset of the pandemic in early 2020, much research has been devoted to the statistical estimation of COVID-19 parameters for models similar to the one we propose in equation (2.2). Maximum likelihood and least square estimates may be used to fit the parameters to observed time series, such as in [11]. Alternative approaches frame the calibration problem within a Bayesian framework, which is arguably more natural to handle the uncertainties associated with those estimates, see [12]. Whist the exploration of sound methodologies to calibrate our model was considered during the study group, due to time constraints we decided to concentrate our efforts on how to use model (2.2) to explore worldwide equitable vaccinations strategies. We have therefore employed the available literature and further considerations to inform the values of our model parameters, as discussed below.

We present here numerical simulations for the case of three interconnected countries, where the following parameters are used:

$$
\gamma=1 / 14, \quad \boldsymbol{\beta}=\left[\begin{array}{ccc}
1.6 & 0.05 & 0.05 \\
0.05 & 1.6 & 0.05 \\
0.05 & 0.05 & 1.6
\end{array}\right] \gamma, \quad N=\left[\begin{array}{c}
6 \\
3.6 \\
3
\end{array}\right] \times 10^{8}
$$

We chose the value of $\gamma$ based on the commonly stated guideline that COVID-19 symptoms last for around two weeks. The intra-country infection rates, $\beta_{i i}$, are chosen to yield a basic reproduction number (number of secondary infections per infected indi- 
vidual) $R_{0}=\beta / \gamma=1.6$. Note that COVID-19 estimates of $R_{0}$ vary significantly in the literature. [13] reviews a series of works whose estimates for $R_{0}$ in Wuhan during January 2020 are between 1.4 and 6.5 . In fact, the value of $R_{0}$ in a country varies with time, and is reduced by external interventions such as lockdowns or social distancing. We however model it as constant in this report for simplicity. Finally, values of $N_{i}$ in equation (2.3) are chosen to represent the approximate populations of Southeast Asia, Western Europe and the United States respectively, as done in [14].

Figure 2 simulates the development of the pandemic in the three above regions, under three different functional forms of the vaccination rates $\xi_{i}(t)$ in equation (2.2). Notice how the total number of infected individuals, shown above each plot, varies significantly according to the chosen vaccination rate. In particular, the SIRV model seems to suggest that the rate of vaccination in the early stages of the epidemic, such as the first 3-4 months, is crucial to reduce the number of overall infections experienced by a country.

\section{Cost of the Epidemic to Countries}

The aim of this section is to use the model presented in equation (2.2) to quantify the costs that countries face to overcome the epidemic. This will set the basis to investigate (equitable) strategies of vaccine allocation in upcoming sections.

In model (2.2) we consider as given inputs (i.e., inputs we have no control over) the initial conditions for the variables $S, I, R, V$, the value of the recovery rate $\gamma$, the interand intra- country infection rates $\beta_{i j}$ and the country populations $N_{i}$. We assume all of these to be time-independent for simplicity, although this assumption could of course be relaxed if necessary. The only other inputs to the model are the country vaccination rates $\xi_{i}(t)$ : these we do assume to have some control of, in order to explore different vaccination strategies.

The aim is therefore to determine values of the vaccination rates $\xi_{i}$ which achieve some apparent best result, under suitable constraints. To give mathematical meaning to the concept of best result, we introduce a cost function for each country, $C_{i}$. This should quantify the social and/or financial cost that country $i$ experiences as a result of the pandemic and of the vaccination strategies of all countries ${ }^{1}$. Possible types of cost to include in $C_{i}$ are the following:

(1) the economic/social cost to the country of people becoming ill, being treated in hospital, dying;

(2) the cost to the country of buying the vaccine and rolling out the vaccination campaign.

We call the former "social cost" and the latter "vaccination cost". During the course of the study group, we agreed on modelling the social cost for country $i, C_{i}^{\text {soc }}$, as a functional of the number of infected people $I_{i}(t)$ in the country, and the vaccination cost, $C_{i}^{\text {vacc }}$, as a functional of the vaccination rate $\xi_{i}(t)$. We therefore modelled the overall cost to

1 Recall that a non-zero $\beta_{i j}$, the infection rate from country $j$ to country $i$, implies that country $i$ also benefits from improved vaccination in country $j$. 


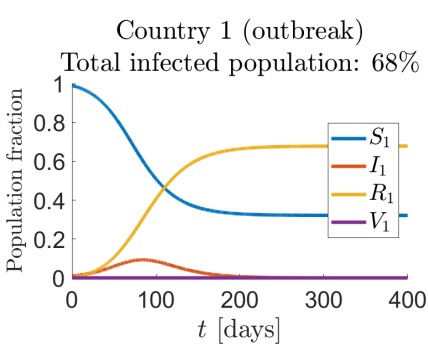

Country 1 (outbreak)

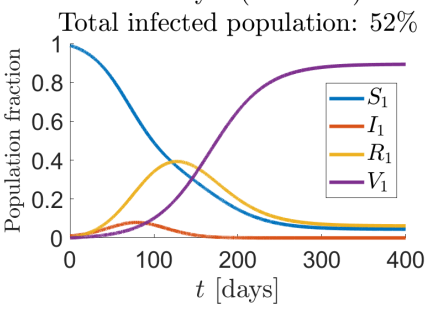

Country 1 (outbreak)

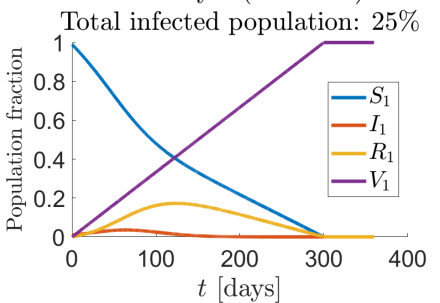

Country 2

Total infected population: $70 \%$

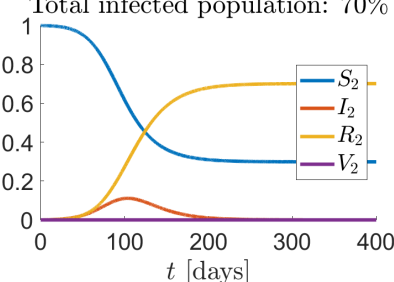

(a)

Country 2

Total infected population: $49 \%$

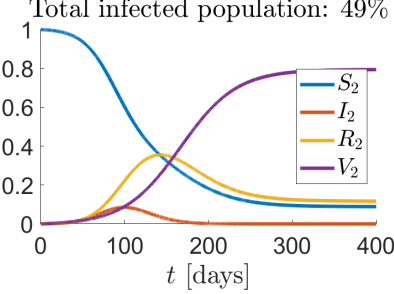

(b)

Country 2

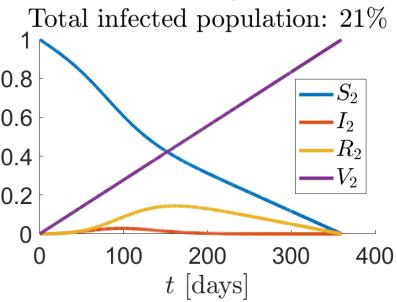

(c)
Country 3

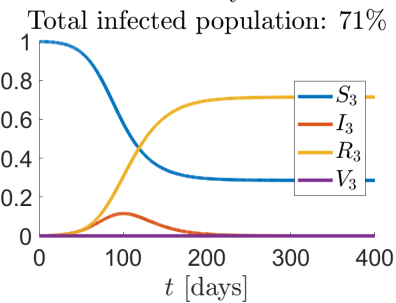

Country 3

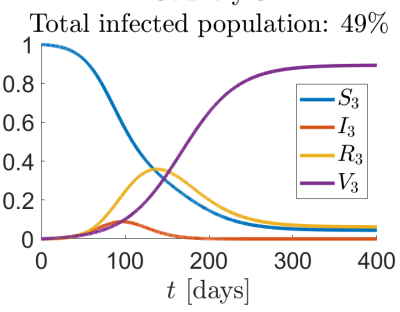

Country 3

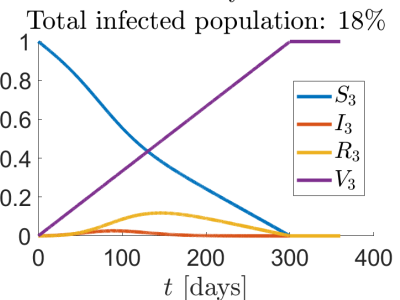

Figure 2. Development of an epidemic according to the SIRV model presented in equation (2.2), with parameters as in (2.3). Country 1 is the origin of the outbreak with $1 \%$ of its population infected initially. The per-country percentage of infected population at the end of the epidemic is reported above each plot. Plots in different rows refer to a different functional form of the countries' vaccination rates. (a) No vaccination takes place in any of the countries, $\xi_{i}(t)=0$ for $i=1,2,3$. (b) $\xi(t)$ is chosen to model initial delay in vaccination rollout and asymptotic limit of proportion of vaccinated population. Specifically, $\xi_{i}(t)=c_{i} \sinh ^{2}[0.015 t-2.5]$, where $c_{i}$ is chosen such that $90 \%$ of the population eventually becomes vaccinated in Countries 1 and 3 , and $80 \%$ of the population receives the vaccine in Country 2. (c) Vaccination rate is constant until the entire population is vaccinated: $\xi_{1}(t)=2 \times 10^{6}, \xi_{2}(t)=1 \times 10^{6}, \xi_{3}(t)=1 \times 10^{6}[\text { day }]^{-1}$.

country $i$ as sum of the two:

$$
C_{i}\left[I_{i}, \xi_{i}\right]=C_{i}^{\mathrm{soc}}\left[I_{i}\right]+C_{i}^{\mathrm{vacc}}\left[\xi_{i}\right]
$$

The square brackets in (3.1) are meant to be a reminder that the different costs are functionals, i.e. their arguments are functions (of time, in our case).

A natural option for $C_{i}^{\text {soc }}$ is to have it proportional to the total number of infections 
experienced by country $i$ during the pandemic. Such a view, however, would not account for the fact that, once the number of infected people gets too high and hospitals fulfil their bed capacity, the cost (especially in terms of lost lives) might rise steeply. To account for this, we instead define the social cost to country $i$ as follows:

$$
C_{i}^{\mathrm{soc}}\left[I_{i}\right]=\int_{0}^{T} U\left(I_{i}(t)\right) d t, \quad T>0,
$$

where the utility function $U$ is the identity function up to some threshold $\lambda_{i}$ (the country's bed capacity), and it is still linear but with an increased slope after $\lambda_{i}$ :

$$
U(x)=\left\{\begin{array}{cl}
x & x \leq \lambda_{i} \\
x+b_{i}\left(x-\lambda_{i}\right) & x \geq \lambda_{i}
\end{array} .\right.
$$

The slope of the utility function after $x=\lambda_{i}$ is therefore equal to $1+b_{i}, b_{i} \geq 0$. We choose $\lambda_{i}=0.05 N_{i}$ and $b_{i}=1$ in the simulations shown in this report. The time horizon $T>0$ in equation (3.2) will be chosen large enough in our simulations for the epidemic to be considered overcome after time $T$ (i.e., $I_{i}(t) \approx 0$ for $\left.t>T\right)$.

As to the form of the function $C^{\text {vacc }}$, the simplest guess would probably be to have it proportional to the total number of vaccines that country $i$ buys and administers to its own population, that is $C_{i}^{\text {vacc }} \propto \int \xi_{i}(t) d t$. There is however a problem with such a choice: if the model is allowed to run to the end, then everyone ends up being vaccinated and the total cost will be always the same, independently of the choice of $\xi_{i}$ (provided $\xi_{i} \neq 0$, of course). Furthermore, in the real world, there is not just a fixed cost per vaccine. Much of the expenditure goes for example into upfront costs, such as building factories or preparing vaccination hubs. Similar costs will increase with higher vaccination rates. In light of this, and while acknowledging that the following is anyway a modelling simplification, we decided to consider a vaccination cost directly proportional to the maximum rate of vaccine administration:

$$
C_{i}^{\mathrm{vacc}}\left[\xi_{i}\right]=k_{i} \max \left\{\xi_{i}(t) \mid t \geq 0\right\} .
$$

The role of $k_{i}$ in equation (3.4) is to distinguish between the financial abilities of different countries: $k_{i}$ will be larger in countries which can hardly afford to purchase and administer the vaccine, and smaller in richer countries. We refer to $k_{i}$ as to the effective vaccination cost parameter for country $i$. Values of $k_{i}$ will be chosen to explore a range of behaviours in section 5 and section 6 .

Equations (3.1)-(3.4) define the costs we use. In order to explore worldwide vaccination strategies, then, we can:

(1) Optimise the global cost, $\sum_{i} C_{i}$. In other words, find vaccination rates $\xi_{i}(t)$ which minimise this global cost.

(2) Use a game theoretic approach, where each country separately attempts to minimise its own cost function $C_{i}$. In this context, besides looking for e.g. Nash equilibria, one may also consider a coordinating contract for countries to follow and under which the outcome of each country following its own best strategy would coincide with the global minimum of point 1 (see [14] for a similar idea in the context of influenza). 


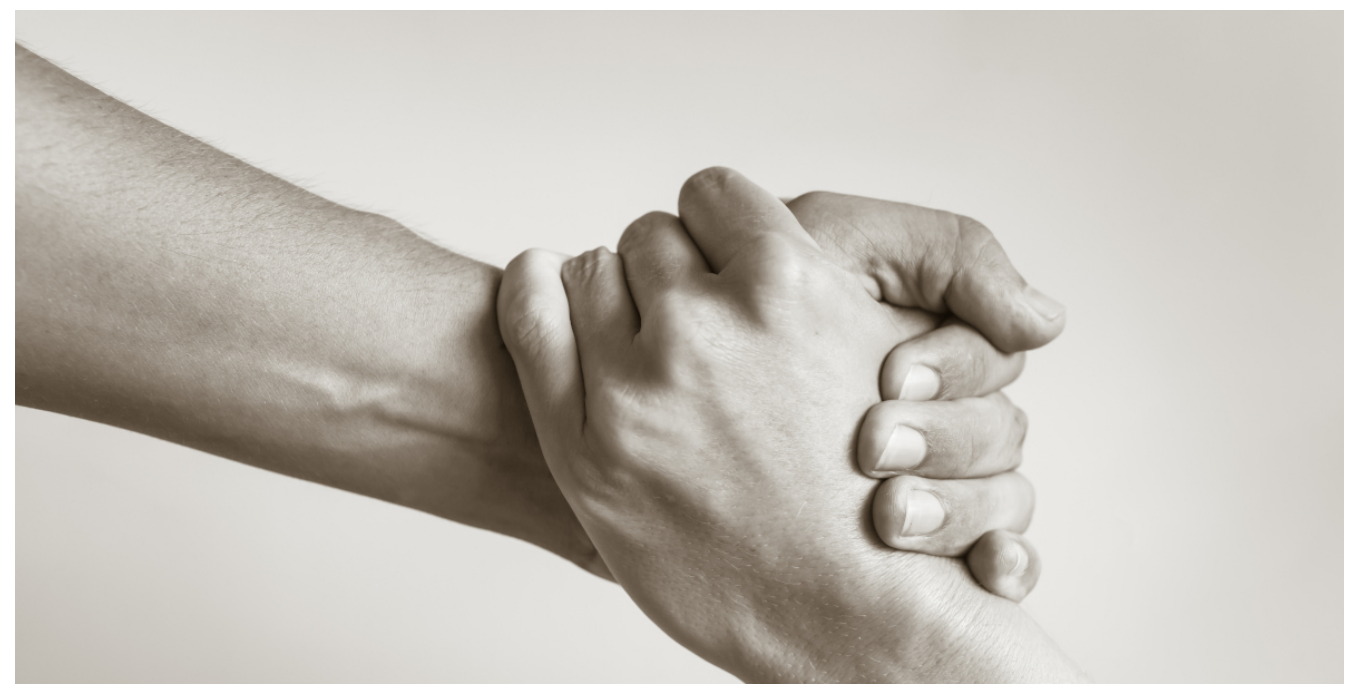

Figure 3. By paying for the vaccination costs of countries which cannot afford them, wealthier countries may ensure that vaccines do not become a privilege for the few and that the pandemic is fought effectively worldwide.

Details and numerical simulations regarding approach 1 are discussed in section 5, while the game theoretic approach is expanded upon in section 6 .

As a final step before looking at numerical results, however, we wish to discuss how to include in our framework the possibility that wealthier countries help countries in financial need, e.g. by donating part of their own vaccines to them and/or by taking on part of their vaccination costs. We explain this in section 4 .

\section{Helping out Countries in Need}

One of the team's aims during the collaborative study group work has been to perform a preliminary investigation of the potential advantage in countries donating vaccine to other countries. In this regard, a recent call for global equitable access to COVID-19 vaccines comes from the COVAX project. The project aims to reduce the tragic loss of life around the world and to improve the recovery of the global economy. We propose in the following a simple framework to model the possibility of trans-national vaccine "donation", which is straightforward to include when optimising costs such as the ones proposed in section 3 .

In the framework we are going to discuss, we assume that country $i$ buys a certain amount of vaccine, keeps some (presumably most) for itself and donates the rest freely to one or more other countries ${ }^{2}$. We call $D_{i j}(t)$ the rate at which country $i$ donates vaccine to country $j$ and assume that country $j$ administers such vaccine doses to its own population. We include the information of vaccine donations in the off-diagonal

2 See for example [5], a recent letter from the director of the Wellcome Trust [link] to the UK newspaper The Guardian, urging leaders to donate vaccines. 
elements of the matrix $D$ :

$$
D=\left(D_{i j}\right) \in \mathbb{R}^{M \times M} .
$$

The diagonal elements of $D$ instead represent the rate (number per day) of vaccines that each country keeps for itself. We thus have that:

- The rate at which country $i$ buys vaccine is $\hat{\xi}_{i}=\sum_{j} D_{i j}$;

- The rate at which country $j$ rolls out vaccines to its own population is $\xi_{j}=\sum_{i} D_{i j}$.

This means that, in a donation setting, the relevant variable in defining the vaccination $\operatorname{cost} C_{i}^{\text {vacc }}$ for country $i$ becomes $\hat{\xi}_{i}$, rather than $\xi_{i}$. The expression in equation (3.4) is thus replaced by:

$$
C_{i}^{\operatorname{vacc}}\left[\hat{\xi}_{i}\right]=k_{i} \max \left\{\hat{\xi}_{i}(t) \mid t \geq 0\right\} .
$$

Apart from the above change, the setting outlined in section 3 to define cost functions remains unaltered when donations are included. The independent variables in our model (equation (2.2) and cost functions) become the rates $D_{i j}$, which can be optimised with respect to the cost function or to analyse countries' strategies in a game theoretic fashion. Note that the framework presented in this section is in fact an extension of the one of section 3: in a non-donating model, the matrix $D$ is diagonal, hence $\hat{\xi}_{i}=\xi_{i}$ for all $i$, and the expression in equation (4.2) recovers the one in (3.4).

\section{Minimising Global Costs}

In this section we address the problem of choosing vaccination rates $\xi_{i}(t)$ which minimise the global cost of the pandemic. Note that, if the donation framework is included in addressing this problem, then constraints on the amount/percentage of vaccines that each country is willing to donate become necessary. Otherwise, any optimal solution will involve the country with the lowest $k_{i}$ to buy vaccines for all other countries. During the limited time of the study group, we were unfortunately unable to carry out numerical experiments with different constraints on each country's willingness to donate. We therefore show here results where the cost functions are as presented in section 3 (i.e., no donations) and will include the donation framework in the upcoming section 6 .

The cost we consider here is the overall cost worldwide:

$$
C=\sum_{i} C_{i}\left[I_{i}, \xi_{i}\right]
$$

where $C_{i}$ is defined in equation (3.1). We assume the perspective of a hypothetical central authority, which obtains vaccines from some unspecified source and decides how to distribute them among countries so that the cost in (5.1) is minimised. Note that the only independent variables are here the vaccination rates $\xi_{i}: I_{i}$ is itself function of the $\xi_{j}$ 's, see equation (2.2).

From a purely mathematical point of view, we do not need to impose constraints on the $\xi_{i}$ (besides the obvious one of them being non-negative) when minimising the cost $C$ : if vaccination rates get too high, the vaccination component $C_{i}^{\text {vacc }}$ of the cost in equation (3.1) will itself increase with no upper bound. However, in order to be more realistic in our simulations, we suppose that a fixed amount $\Xi$ of vaccines is available 
daily, and formulate our problem as follows:

$$
\begin{gathered}
\min _{\xi \in C_{p}([0, T])} \sum_{i} C_{i}\left[I_{i}, \xi_{i}\right], \\
\text { subject to } \sum_{i} \xi_{i}(t) \leq \Xi \forall t \geq 0 .
\end{gathered}
$$

The space $C_{p}([0, T])$ is the one of piecewise continuous functions over the interval $[0, T]$. Note that, if we were to define the cost to country $i$ as only the social cost, then constraint $(5.2 \mathrm{~b})$ would be necessary as otherwise the optimum would arise from the impractical solution of vaccinating everyone immediately.

One way to approach problem (5.2) is to use optimal control theory. Typically, this means using either Pontryagin's Maximum Principle or the Hamilton-Jacobi-Bellman equation to solve problem (5.2) numerically. These methods, however, require a higher regularity than the one we suppose above and were not easy to implement in the limited amount of time we had. For these reasons, we chose instead the following approach.

We look at a subspace of $C_{p}([0, T])$, which we parameterise with a finite number of parameters. This turns the infinite dimensional control problem into an optimisation problem over a finite dimensional parameter space. We carry out numerical simulations in the case of $M=3$ countries, with parameters as in (2.3), and piecewise constant vaccination rates:

$$
\xi_{i}(t)= \begin{cases}\xi_{i, 1} & t<50 \\ \xi_{i, 2} & 50 \leq t<100 \\ \xi_{i, 3} & t \geq 100\end{cases}
$$

Time is measured in days. We seek to minimise the total cost $C$ by optimising over the 9 parameters $\xi_{i, j}, 1 \leq i, j \leq 3$. For a first quick implementation, we use Matlab's fmincon built-in routine to find the optimal solution, which uses an interior point method by default.

Figure 4 illustrates the solution to the optimisation problem for different choices of the initial guess where the optimisation starts from. The results show that the problem is numerically unstable, as the optimum found by fmincon is highly dependent on the initial guess. The optimisation algorithm is prone to be attracted to local minima, whose values are fairly different from one another (see caption). This suggests that our problem is highly non-convex, and it remains to be seen how a global minimum can be found efficiently in this case.

One may also wish to restrict the allowed space of rates $\xi_{i}(t)$ to a more regular space. So, let $\varphi_{1}(t), \ldots, \varphi_{k}(t)$ be pre-specified continuous or even differentiable functions of $t$, and let $V=\operatorname{span}\left\{\varphi_{1}, \ldots, \varphi_{k}\right\}$. We can then minimise costs in $V$. If $\xi_{i}(t) \in V$, then $\xi_{i}(t)=\sum_{\ell} a_{i \ell} \varphi_{\ell}(t)$ and the optimisation problem (5.2) can be rewritten as follows:

$$
\begin{array}{cl}
\min _{a_{i \ell}} & \sum_{i} C_{i}[I(t), \xi(t)], \\
\text { subject to } & \sum_{i} \sum_{\ell} a_{i \ell} \varphi_{\ell}(t) \leq \Xi \quad \forall t \geq 0 .
\end{array}
$$

We note that the aforementioned optimisation problem of fitting piecewise constant $\xi_{i}(t)$ is fundamentally different as it allows for non-smoothness in the constraint function 

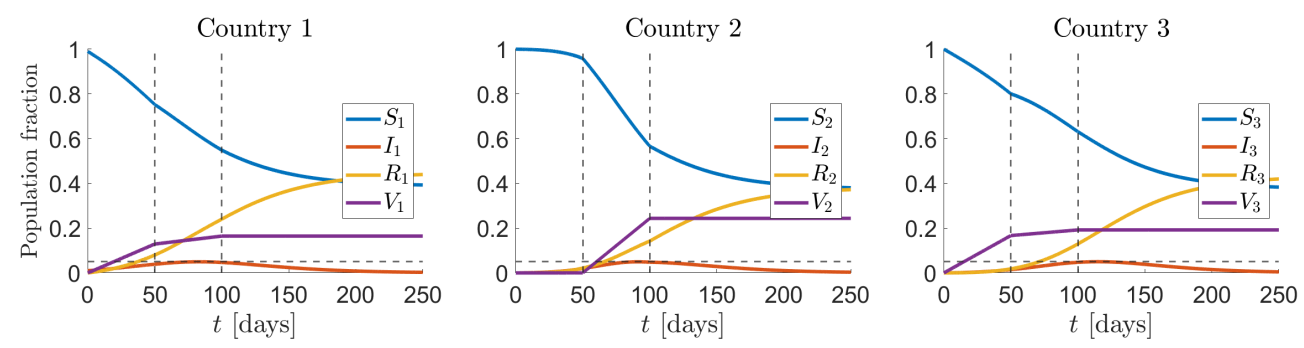

(a)
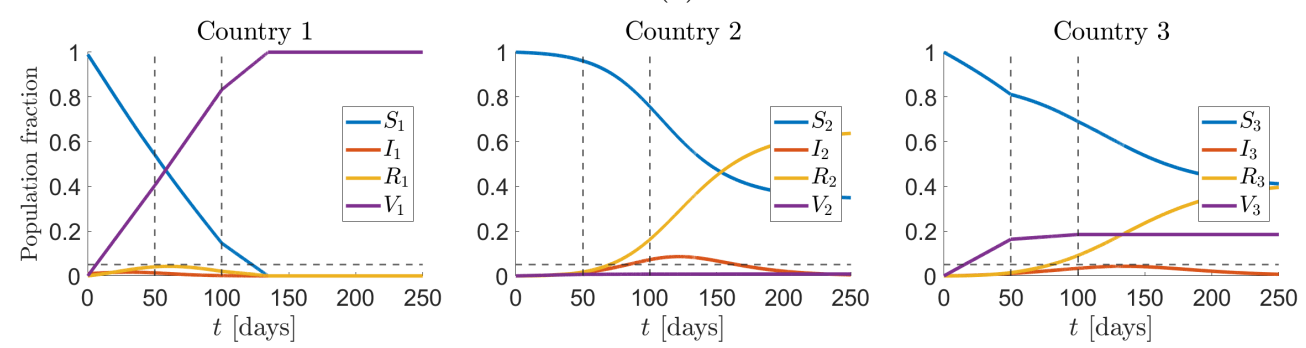

(b)
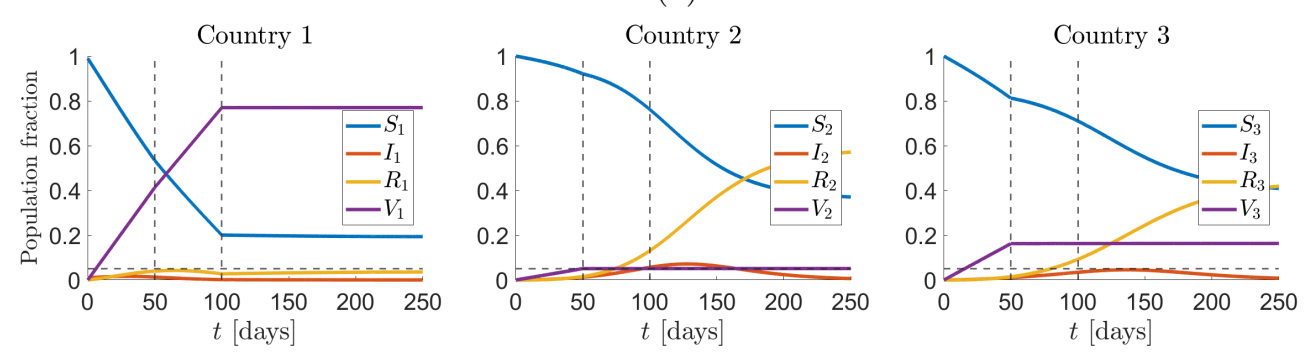

(c)
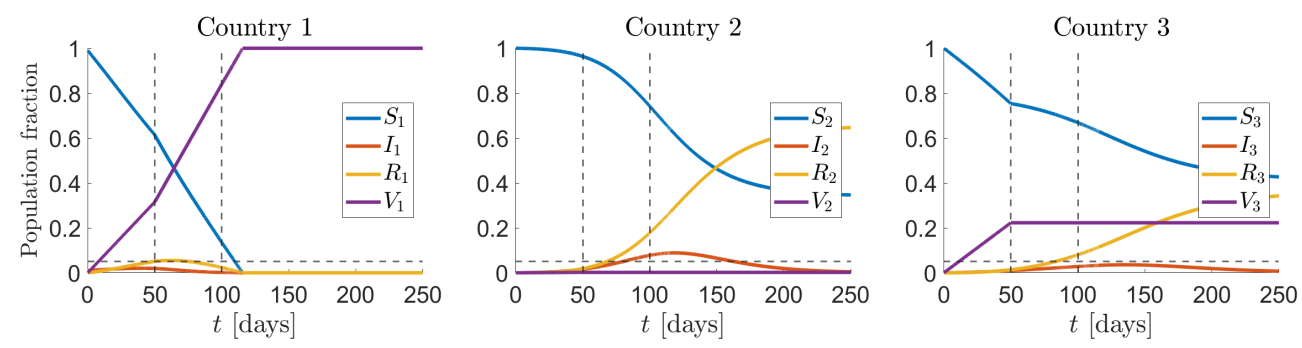

(d)

Figure 4. Solutions to the optimisation problem (5.2) using piecewise constant vaccination rates $\xi_{i}$ as in (5.3). Each row shows the result associated with a different initial condition for the minimisation algorithm. The minimisation algorithms tries to keep country infections below hospital bed capacity (dashed horizontal lines), see equation (3.3). Model parameters as in (2.3), with country 1 being the source of the outbreak with $1 \%$ infected population initially. Maximum vaccination rate is $\Xi=N / 200[\text { day }]^{-1}$, see $(5.2 \mathrm{~b})$. The effective vaccination cost parameter is $k_{i}=100$ for all countries. The figure highlights the sensitivity of the numerical optimisation (Matlab's fmincon solver is used) to different initial guesses of the minimising point. (a) Initial guess is a constant vaccination rate allowing countries to vaccinate their population within 200 days. (b) Initial guess in (a) is perturbed by less than $0.4 \%$ on each parameter. (c) Initial guess in (a) is perturbed by up to $4 \%$ on each parameter. (d) Initial guess is randomly chosen from a uniform distribution. Dimensionless costs of the objective: (a) 320, (b) 796, (C) 655, (d) 806 . 
and thus could lead to a very different behaviour of the optimisation solver. We leave the problem presented in equations (5.4a)-(5.4b) as topic of future investigation.

\section{Game Theory Perspective}

In the framework presented so far, each country can invest resources to increase its own vaccination rate (and other's too, in a donating framework), thus reducing its social cost while increasing the economic cost of the vaccination campaign. The discussion in section 5 assumes that a central authority exists, which decides how to distribute the available vaccines to minimise overall global costs. An alternative, and in fact more realistic, approach is the one where countries aim to minimise their own cost by optimising their own vaccine strategy. If country $i$ knows in advance the infection and vaccination rates of all other countries, then the vaccination rate $\xi_{i}^{O}$ can be considered optimal for country $i$ if:

$$
C_{i}\left[\xi_{1}, \ldots, \xi_{i}^{O}, \cdots, \xi_{M}\right]=\min _{\xi_{i}} C_{i}\left[\xi_{1}, \cdots, \xi_{i}, \ldots, \xi_{M}\right]
$$

A more realistic situation is however the one where country $i$ knows all countries' infection rates but does not know the vaccination rate that other countries will adopt. In this context we can analyse different kinds of optima, using the tools of game theory.

A vaccination strategy $\boldsymbol{\xi}^{\mathrm{N}}=\left(\xi_{1}^{\mathrm{N}}, \ldots, \xi_{M}^{\mathrm{N}}\right)$ is a Nash equilibrium if:

$$
C_{i}\left[\xi_{1}^{\mathrm{N}}, \ldots, \xi^{\mathrm{N}}, \cdots, \xi_{M}^{\mathrm{N}}\right]=\min _{\xi_{i}} C_{i}\left[\xi_{1}^{\mathrm{N}}, \cdots, \xi_{i}, \ldots, \xi_{M}^{\mathrm{N}}\right] \quad \forall i
$$

In other words, $\xi^{\mathrm{N}}$ is a Nash equilibrium if no country can be better off by choosing a strategy other than the one prescribed to it by $\boldsymbol{\xi}^{\mathrm{N}}$, assuming all other countries follow $\boldsymbol{\xi}^{\mathrm{N}}$. If a Nash equilibrium exists and it is unique then it represents the best strategy a country can adopt, assuming all other countries are rational and will chose their best strategy. If there are no or more than one Nash equilibria, then more advanced game theory tools are needed for the system analysis.

On the other side, we may define a vaccination strategy $\boldsymbol{\xi}^{\mathrm{SO}}$ as system-optimal (SO) if it minimises the global cost over all possible vaccination strategies $\boldsymbol{\xi}$ :

$$
\sum_{i} C_{i}\left[\boldsymbol{\xi}^{\mathrm{SO}}\right]=\min _{\boldsymbol{\xi}}\left(\sum_{i} C_{i}[\boldsymbol{\xi}]\right)
$$

Note that this is the approach followed in section 5. Of course, strategies which represent Nash equilibria may not be SO strategies, and vice-versa.

We now compare Nash equilibria and SO strategies in the case of two countries $(M=2)$ and vaccination rates $\xi_{i}$ constant in time. This leaves us with only two free parameters in the optimisation problem, which also makes it easy to visualize the solution. Values of the epidemiological and population parameters for the two countries, as defined in model (2.2), are as follows:

$$
\gamma=1 / 14, \quad \boldsymbol{\beta}=\left[\begin{array}{cc}
1.6 & 0 \\
1.3 & 1.6
\end{array}\right] \gamma, \quad N=\left[\begin{array}{l}
6 \\
3
\end{array}\right] \times 10^{8} .
$$

We suppose that country 1 is the source of the epidemic with $1 \%$ infected population 

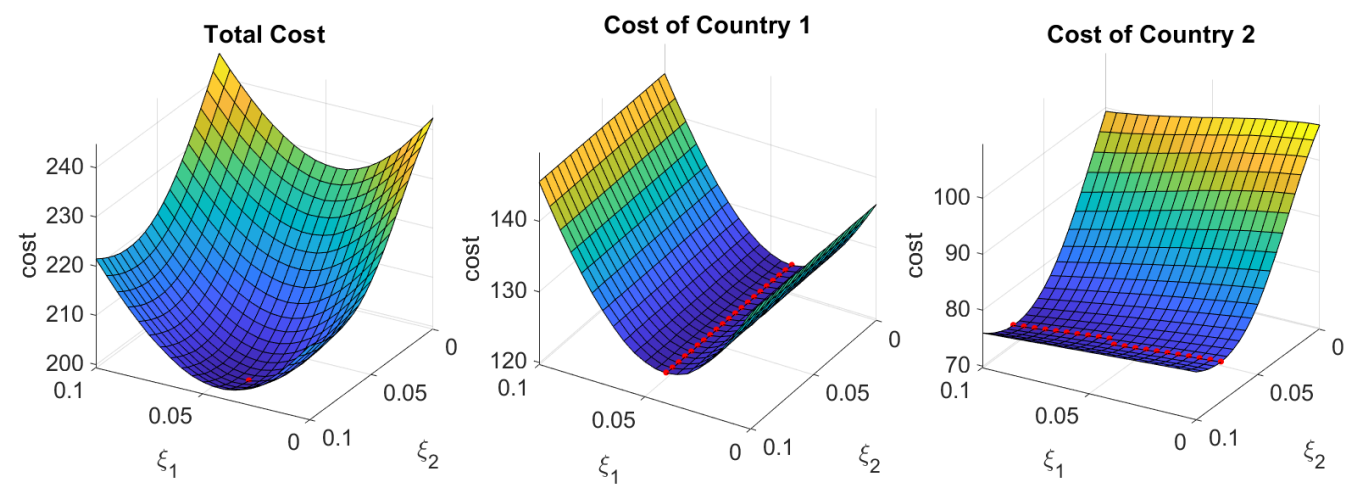

Figure 5. Cost functions for two countries with comparable vaccination costs $\left(k_{1}=\right.$ $\left.1000, k_{2}=500\right)$ and model parameters as in (6.4). On the plot to the left, the total cost for the two countries is shown as a function of the constant vaccination rates $\xi_{1}$ and $\xi_{2}\left(\xi_{i}\right.$ units: $10^{8} /$ day $)$. The global minimum is near $\left(\xi_{1}, \xi_{2}\right) \approx(0.04,0.085)$, highlighted in red. Each country's cost is plotted next, with red dots marking the optimal selfish decision for each country. Observe that the best decision of country 1 is unaffected by country 2 , since we assume there is no disease transmission from country 2 to $1\left(\beta_{12}=0\right)$. Country 1 will then choose $\xi_{1} \approx 0.04$ (second plot). Country 2 , in turn, will choose $\xi_{2} \approx 0.08$ (last plot), yielding a Nash equilibrium which is close to the above global optimum.

initially, while there are no infections in country 2 originally. As shown above, however, infections are transmitted from country 1 to country 2 at a rate $\beta_{21}=1.3 \gamma$.

Figure 5 shows the costs to each of the two countries as well as the total cost, as functions of the two vaccination rates $\xi_{1}$ and $\xi_{2}$. In this simulation we imagine that the effective vaccination costs to the two countries are not too dissimilar $\left(k_{1}=1000\right.$ and $k_{2}=500$, i.e. country 2 is a bit richer than country 1$)$. What the figure shows is that, in this case, each player's selfish strategy would produce an equilibrium close to (but not exactly equal to) the system's optimum.

Let us now suppose that country 2 has a substantially stronger economy than country $1\left(k_{1}=400, k_{2}=100\right)$ and allow country 2 to donate part of their vaccines to country 1 , through the framework presented in section 4 . In terms of the matrix $D \in \mathbb{R}^{2 \times 2}$ in equation (4.1), we consider $D_{11}$ and $D_{21}$ as free variables (each allowed to range between 0 and $0.1 \times 10^{8}[\text { day }]^{-1}$ ) and impose:

$$
D_{12}=0 \quad \text { and } \quad D_{22}=0.1-D_{21} .
$$

This means that the richer country 2 will buy vaccines at a constant rate $D_{21}+D_{22}=0.1$, possibly donating some $\left(D_{21}\right)$ to country 1 , while the latter will buy vaccines at a rate of at most 0.1 and will keep them all for itself.

Figure 6 shows that, in this case, we have a discrepancy between the system-optimal (SO) strategy and the Nash equilibrium. The former, $\left(D_{11}, D_{21}\right) \approx(0.085,0.015)$, is found by minimising the total cost. However, if we take the perspective of country 2 (right-most plot in the figure), for any value of $D_{11}$ its cost is minimised at $D_{21}=0$, where the country makes no donation to country 1 . Accordingly, the best strategy of country 1 is to choose $D_{11}=0.09$. The Nash equilibrium is thus $\left(D_{11}, D_{21}\right) \approx(0.09,0)$, 
Total Cost

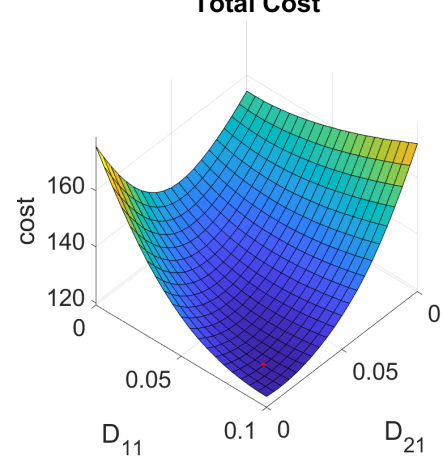

Cost of Country 1

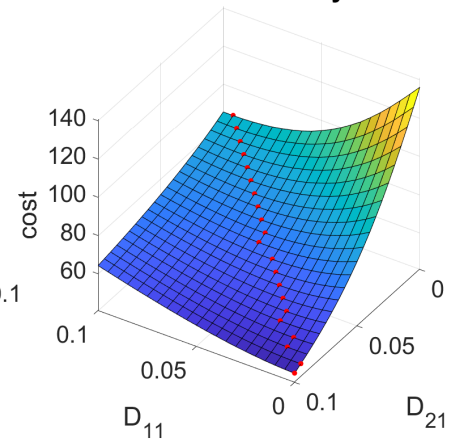

Cost of Country 2

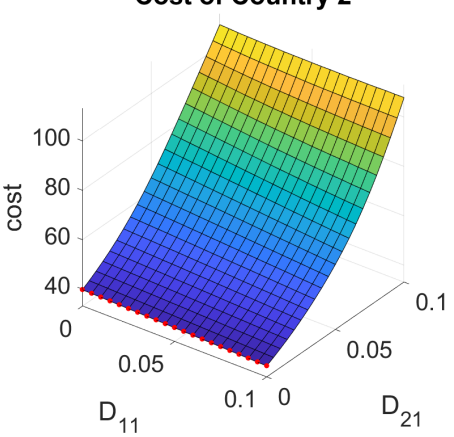

Figure 6. Costs for two countries as functions of $D_{11}$ and $D_{21}$, with $D_{12}=0, D_{22}=$ $0.1-D_{21}$ and $k_{1}=400, k_{2}=100$. Units for $D_{i j}: 10^{8}$ [day $^{-1}$. In this setting, country 2 buys vaccines at a rate of $D_{21}+D_{22}=0.1$, of which $D_{21}$ are donated to country 1 . The two countries' vaccination rates are therefore $\xi_{1}=D_{11}+D_{21}$ and $\xi_{2}=D_{22}$. The plot on the left shows that the system-optimal strategy is $\left(D_{11}, D_{21}\right) \approx(0.085,0.015)$. In this case, country 1 vaccinates at a rate of 0.1 with $15 \%$ of the cost born by country 2 . However, from country 2's selfish perspective (last plot), the best value of $D_{21}$ for any $D_{11}$ would be $D_{21}=0$ (i.e., not making a donation). Accordingly, country 1 would then vaccinate at a lower rate of 0.09 , bearing its own full financial cost. The Nash equilibrium is substantially different from the system-optimal strategy.

which differs non-negligibly from the SO strategy: it yields a lower rate of vaccination for country 1 (0.09 rather than 0.1$)$ and a higher financial cost for the same country.

Note that such a conclusion is to be interpreted strictly within the limitations of our model. The latter has been proposed during the limited time allowed by the ESGI 165 duration and it necessarily misses on modelling features which would likely encourage vaccine donation (more on this in the discussion). Since the limited time would not allow us to focus on perfect realisticness, our aim has been to explore model behaviours in a couple of simple examples, which already point out that single countries' best strategies vary significantly according to the approach taken in minimising costs.

Although we have here looked at numerical results for the case of two countries only, the general setting would not change in the case of $M \geq 3$ countries. The strategy of country $i$ would be represented by the $i$ th row of $D, D^{i}=\left(D_{i 1}, \ldots, D_{i M}\right)$, and the Nash equilibrium would be given by the matrix $D^{*}=\left(D^{* 1} ; \ldots ; D^{* M}\right)$ such that

$$
C_{i}\left[\boldsymbol{D}^{*}\right]=\min _{D^{i}}\left(C_{i}\left[D^{* 1} ; \ldots ; D^{i} ; \ldots ; D^{* M}\right]\right) .
$$

As we have already seen for $M=2$, the Nash equilibrium may be very different from the system's optimum. It would be interesting to explore numerically both this and the non-donating model, comparing how much a country can gain by adopting the donating strategy in different scenarios. Unfortunately, we could not explore this during the limited time of the study group. Qualitatively, however, we expect that the Nash equilibria of the two models will be very similar when inter-country transmission is very low $\left(\beta_{i j} \ll 1\right)$. In this case, within our framework, richer countries will have little incentive to pay for 
other countries' vaccination costs. For higher values of $\beta$ new interesting solutions should instead emerge.

\section{Discussion and Future Work}

In this report, we have presented the results of the work carried out by our team during the ESGI 165 study group, between the 12th and the 16th of April 2021. With reference to the current COVID-19 pandemic, the aim of the team has been to propose a mathematical framework allowing to investigate "best" international strategies of vaccination rollout, in order to fight the pandemic effectively at a global scale.

To approach the problem, we have made use of a compartmental model (SIRV) based on ordinary differential equations. The model simulates the spread of an epidemic, both within and between countries, while these carry out vaccination campaigns. To give mathematical meaning to the concept of "best strategy", for any country we have defined a cost function, accounting for both the social costs (number of infections, exceeding bed capacity) and the financial costs (vaccination campaign) that the country incurs as a consequence of its epidemiological situation and vaccination strategy. A parameter in our model allows to distinguish between the financial strength of different countries.

We have then investigated two possible strategies into minimising costs. A first one where a central authority decides how to distribute the available vaccines to the different countries, in order for the global cost to be minimised. A second one where instead, as in a competitive game, each country aims to minimise its own cost, which is however partially dependent on all other countries' vaccination strategies (e.g., increasing vaccination in country $i$ will lower the transmission from $i$ to $j$ and will therefore lower the social cost of country $j$ ). Moreover, we have extended our modelling framework to allow for the possibility that countries donate part of the vaccines they have paid for to other countries. This is meant to mimic the dynamics of current international programmes such as COVAX, and investigate the gain that its implementation may have both globally and at the single-country level.

In order to investigate results numerically, we have considered either piecewise constant or constant vaccination rates in each country. In the case of piecewise constant rates, our simulations show that system-optimal strategies may be difficult to find numerically, since the optimisation algorithm gets trapped in local minima. We however suspect that the numerical instability may be overcome by using a subspace of more regular functions. In the simple case of two countries, and with constant vaccination rates, we have shown that the two approaches mentioned at the beginning of the section lead to different results. System-optimal strategies and Nash equilibria may not coincide, and the extent to which a richer country gains from donating part of their vaccines is likely to depend on which perspective is being considered.

We wish to stress that our results are not meant to be ground for actual decision making. The framework we have presented here is at an early stage of development, and is currently built around a simple toy model (the SIRV) which certainly misses to capture several intricacies related to COVID-19 transmission/development and vaccination. Any conclusion reached is only to be interpreted within the confines of our model, which for example does not include virus variants and the waning effect of immunisation over 
time. We expect, for instance, that accounting for similar aspects would increase the advantage to wealthier countries of ensuring that infections are kept low also outside their own borders, and would therefore encourage vaccine donation. In the following we share some of the ideas developed during the study group collaboration to make our modelling framework more realistic and to merge political decisions within it.

First, the number of compartments and the dynamics itself of the SIRV model may be modified to account for the fact that, among the infected group, symptomatic individuals should not be vaccinated while some of the asymptomatic ones would be. Moreover, it would be interesting to consider different vaccines, which could be distinguished by their price and efficacy. As mentioned above, immunity (either due to infection or vaccination) wanes over time. Our model could therefore include one or more parameters that allow for reinfection in the recovered and vaccinated groups. In this regard, we stress that these and other parameters, such as the percentage of asymptomatic individuals who get vaccinated, could be included in our model in the form of random variables, possibly within a Bayesian framework, to better account for the uncertainties that surround their estimation. A more realistic modelling might also be characterised by time dependent intra- and inter- country transmission rates, which are affected by political decisions such as national lockdowns and international travel bans.

Finally, we note that our model assumes the existence of a supply chain which delivers vaccines at a constant rate. In periods of high demand, however, ensuring its resilience is no simple task: several links compose the chain (from raw materials to vaccine producers, packaging, distribution to governments, actual allocation to the population, etc., see [15]), so disruption at any point may cause shortages or delay, [16]. At the start of the study group, the team considered the possibility of modelling supply chains in order to investigate strategies which would favour their resilience against disruptions. This route was not pursued in the end, but we think the study would naturally complement what is discussed here and we reserve it as a topic of further investigation.

The code developed during the collaboration can be found at the following url: https: //github.com/liuyue002/ESGI165_vaccinesupply.

\section{Acknowledgements}

This project has received funding from the European Union's Horizon 2020 research and innovation programme under the Marie Skłodowska-Curie grant agreement No. 764850 (SAGEX). This is paper SAGEX-21-27.

\section{References}

[1] World Health Organization. Weekly epidemiological update on COVID-19. Technical report, 4 May 2021.

[2] David M Cutler and Lawrence H Summers. The COVID-19 Pandemic and the \$16 Trillion Virus. JAMA, 324(15):1495-1496, 2020.

[3] Stephen R Hanney, Steven Wooding, Jon Sussex, and Jonathan Grant. From COVID-19 research to vaccine application: why might it take 17 months not 17 years 
and what are the wider lessons? Health Research Policy and Systems, 18(61):1-10, 2020.

[4] Carlos del Rio, Gregg Gonsalves, Fatima Hassan, and Matthew Kavanagh. COVID-19: A call for global vaccine equity. The British Medical Journal, March 17, 2021. Open Letter.

[5] Jeremy Farrar. Political leaders must ensure Covid vaccines aren't the preserve of the rich. Guardian newspaper, April 28th, 2021. Available at https://www.theguardian.com/commentisfree/2021/apr/28/ political-leaders-covid-vaccines-rich.

[6] Salim S Abdool Karim. Vaccines and SARS-CoV-2 variants: the urgent need for a correlate of protection. Lancet, 397(10281):1263-1264, 2021.

[7] Giovanni Rezza. Ministero della Salute (Italian Ministry of Health). https://www.trovanorme. salute.gov.it/norme/renderNormsanPdf?anno= 2021\&codLeg=79033\&parte $=1 \% 20 \&$ serie=null, 2021.

[8] CDC. FAQs about COVID-19 Vaccination. https://www. cdc.gov/coronavirus/ 2019-ncov/vaccines/faq.html, 2021.

[9] Florian Krammer, Komal Srivastava, Hala Alshammary, Angela A Amoako, Mahmoud H Awawda, Katherine F Beach, Maria C Bermúdez-González, Dominika A Bielak, Juan M Carreño, Rachel L Chernet, et al. Antibody responses in seropositive persons after a single dose of SARS-CoV-2 mRNA vaccine. New England Journal of Medicine, 384(14):1372-1374, 2021.

[10] Jingjing He, Yifei Guo, Richeng Mao, and Jiming Zhang. Proportion of asymptomatic coronavirus disease 2019: A systematic review and meta-analysis. Journal of medical virology, 93(2):820-830, 2021.

[11] Amir Hossein Amiri Mehra, Mohsen Shafieirad, Zohreh Abbasi, and Iman Zamani. Parameter Estimation and Prediction of COVID-19 Epidemic Turning Point and Ending Time of a Case Study on SIR/SQAIR Epidemic Models. Computational and Mathematical Methods in Medicine, 2020:1-13, 2020.

[12] Daniela Calvetti, Alexander Hoover, Johnie Rose, and Erkki Somersalo. Bayesian dynamical estimation of the parameters of an SE(A)IR COVID-19 spread model, 2020.

[13] Ying Liu, Albert A Gayle, Annelies Wilder-Smith, and Joacim Rocklöv. The reproductive number of COVID-19 is higher compared to SARS coronavirus. Journal of travel medicine, 27, 2020.

[14] Hamed Mamani, Stephen E Chick, and David Simchi-Levi. A game-theoretic model of international influenza vaccination coordination. Management Science, 59(7):1650-1670, 2013.

[15] L. E. Duijzer, W. van Jaarsveld, and R. Dekker. Literature review: The vaccine supply chain. European Journal of Operational Research, 268(1):174-192, 2018.

[16] João Pires Ribeiro and Ana Barbosa-Povoa. Supply Chain Resilience: Definitions and quantitative modelling approaches - A literature review. Computers $\&$ Industrial Engineering, 115:109-122, 2018. 